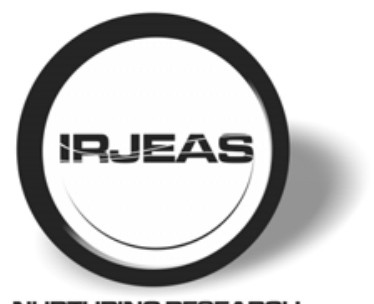

NURTURING RESEARCH
INTERNATIONAL RESEARCH JOURNAL

OF

ENGINEERING \& APPLIED SCIENCES

VOLUME 9 ISSUE 4

ISSN: 2322-0821(0)

ISSN: 2394-9910(P)

Oct 2021 - Dec 2021

www.irjeas.org

\title{
MACHINE LEARNING BASED FAULT DIAGNOSIS SCHEME FOR GRID-CONNECTED PV SYSTEM
}

\author{
*Deepa Singh ${ }^{1}$, Laxman Solankee ${ }^{2}$ \\ ${ }^{1}$ M. Tech Scholar, ${ }^{2}$ Professor \\ , Department of Electrical and Electronics Engineering, Technocrats Institute of Technology, Bhopal, India \\ *Corr. Author - deepa.baghel55@gmail.com
}

DOI - 10.55083/irjeas.2021.v09i04007

\begin{abstract}
Photovoltaic (PV) systems have received a lot of attention in recent decades due to their accessibility and advancements in PV technology. The protection of PV systems from faults such as String to String (SS), String to Ground (SG), Open circuit (OC), and partial shading are the key challenges to the realization of cost-effective and environmentally friendly PV systems. Such unusual circumstances reduce the maximum available $P V$ power. Partial shading and breakdowns in a PV array must therefore be noticed quickly for enhanced system efficiency and reliability. The significant fault current in PV systems can be detected using the existing safety devices in PV systems, such as fuses and residual current detectors. The flowing fault current being of low order is not significant enough for current protection devices to detect if the solar and/or fault mismatch is modest and the fault resistance is high. As a result, under cloudy and low irradiance conditions, the traditional protection devices fail to identify problems, resulting in reliability concerns and photovoltaic fire threats. In this context, a fault diagnosis scheme for $\mathrm{PV}$ systems is presented in this paper, which includes feature extraction using the Discrete wavelet transform, and classification of various defects on the PV system using Decision tree.
\end{abstract}

Keywords- Microgrid, Grid-connected Photovoltaic System, Protection Devices, Machine Learning, Partial Shading, Distributed energy resources (DERs), Decision Tree

\section{INTRODUCTION}

To maintain a secure and continuous power supply, the responsibility to reduce greenhouse gas emissions has resulted in a significantly larger penetration of small and medium-sized renewable resources in distribution networks [1]. Microgrid is a term used to describe a power system network that allows for the connectivity of multiple low-power renewable and/or conventional sources [2]. Due to the intrinsic properties of solar energy, such as simplicity of access, cost-effective operation, and sustainability, PV-based generation has gained great relevance among the most often employed renewable distributed energy resources (DERs) in the microgrid [3]. Despite its widespread adoption, PV's intermittent and irregular working characteristics, as well as its heavy reliance on environmental circumstances, have posed serious hurdles to the protection of $\mathrm{PV}$ integrated microgrids. Under dual-operating modes (gridintegrated and isolated) of the microgrid demand fault resilient operation, the operating circumstances of PV and other DER are more variable, as are the divergent fluctuations in post-fault current-voltage profiles. Aside from line failures, there are a few typical difficulties in the PV array that affect the overall performance of a PV integrated microgrid. Although both line defects and PV system failures are contingencies from which the microgrid must be protected, each type of failure should be handled differently, with a PV fault requiring only that the faulty source be disconnected, whereas line faults require identifying and isolating the faulty line. Traditional relays designed to use current-voltage data, on the other hand, frequently can't tell the difference between problems in the PV array and faults in the distribution line, resulting in inconvenient relay operation and possible power outages.

In recent years, a slew of approaches for protecting microgrids with PV arrays have been presented. Discrete wavelet transformation and decision tree-based methodology [4], as well as superposed Hilbert Transform reactive energy computation [5], are some of the most important methodologies. Time-free transition-based differencing scheme [6], fast recursive discrete fourier transform and fuzzy logic based decision making module for relay current setting [7], discrete wavelet transform and ensemble classifier based scheme [8], DER impedance calculation based technique [9], and differential current based adaptive thresholding [10]. 
International Research Journal of Engineering \& Applied Sciences, IRJEAS

www.irjeas.org, ISSN(O): 2322-0821, Volume 9 Issue 4, Oct-Dec 2021, Page 28-33 DOI - 10.55083/irjeas.2021.v09i04007 Despite the fact that these strategies have been used to deal with a variety of microgrid fault scenarios, none of them have been tested for their ability to distinguish between array and line faults. Techniques for dealing with PV array problems have also been thoroughly documented in the literature [11-14], however all of these strategies have been presented for standalone PV systems and have not been adapted to microgrid systems.

The suggested strategies for protecting photovoltaic integrated microgrids are mostly focused on line or array failures, according to a survey of the literature on the subject. Neither of these methods is capable of detecting and distinguishing the two problems simultaneously. In this context, in addition to performing the intended task of fault detection in microgrid distribution lines, the current work proposes a protection approach for PV integrated microgrids that can also detect faults in the array, preventing nuisance relay tripping and ensuring power supply reliability.

In addition, for the identification and classification of defects in a power system, several computer intelligence approaches such as support vector machine (SVM) [15], decision tree (DT) [4], and fuzzy logic [16] have been used in recent years. The suitability and efficiency of feature extraction and classification methodologies for a computational intelligence-based protection strategy have been thoroughly investigated. The feature extraction method is crucial for preserving the contextual and discriminating information extracted from the source signals. The time, frequency, and mixed time-frequency domain feature extraction approaches may all be generically categorised. For extracting the relevant features, discrete transform fouriers (DFT) [8][17], discrete transform wavelets (DWT), transform

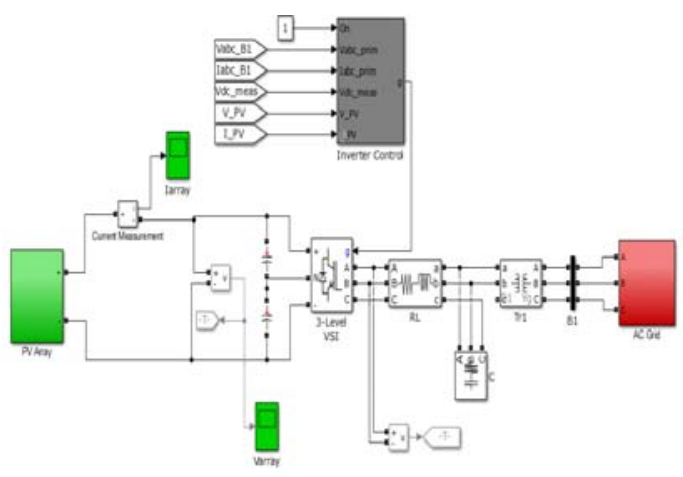

Figure 1: Considered Grid-connected PV System

\section{PROPOSED PROTECTION STRATEGY}

The comparable behaviour of PV array failures and distribution line faults, as seen and explained before,

Hilbert-huang (HHT) [18], and S-transform have been utilized in some of the key protection strategies published in the literature. Pre-processing is required to remove noise and other redundant information before extracting useful and discriminating qualities from the raw input signals.

A protective strategy including fault discrimination in the distribution line and PV array has been adopted in the current study. The proposed approach involves feature extraction using the Discrete wavelet transform and Decision tree for classification of PV array fault. Furthermore, as previously demonstrated, the suggested approach has a high degree of selectivity for carrying out the required action, despite the fact that distribution line faults and PV array faults resembles similar in behaviour.

\section{Grid-connected PV system}

Figure 1 shows the structure of a balanced Gridintegrated $\mathrm{PV}$ system simulated under MATLAB/Simulink environment. The utility Grid is connected to the microgrid through a switch at the point of common coupling (PCC), which allows for the transition between grid-integrated and isolated modes of operation.

In order to analyze the influence of distribution network behaviour during PV array failures, the PV source has been modeled as a distributed series-parallel combination of PV modules. A blocking diode is linked in series with each parallel string to prevent back-up current, and each module contains an anti-parallel bypass diode to avoid hotspot during partial shade.

necessitates an accurate relaying mechanism to discriminate between the two scenarios and avoid the relay from being misoperated. To fulfil the specified duties of protection, a protection method combining a combined framework of Discrete wavelet transform (DWT) and Decision tree based machine learning classifier has been used. The first stage involves utilizing the DWT to extract discriminating qualities from current signals on the relaying bus, followed by Decision tree for categorization of faults using the derived attributes.

\section{A. Feature extraction using Discrete Wavelet Transform (DWT)}

Wavelet transform tools are used to capture and evaluate voltage-current patterns during line faults and disturbances. Wavelet feature extraction techniques extract discriminating features from raw time-domain $\mathrm{dc}$ voltage current profile data and remove all redundant information [8]. The DWT was utilised to extract 
International Research Journal of Engineering \& Applied Sciences, IRJEAS

Www irieas org ISSN(0): 2322-0821, Volume 9 Issue 4, Oct-Dec 2021, Page 28-33

DOI - 10.55083/irjeas.2021.v09i04007 relevant information from time-domain voltage-current data after a fault in this article.

most generally used ensemble approach is bagging, in which the number of subsets is changed by partitioning the full training data using a sampling and substitution procedure. The same learner and a distinct learner train sub-sets.

The signals may be decomposed into several timefrequency domains using DWT. It pulls relevant data in this way, allowing for precise defect detection. Through the mother wavelet's translation shift in time and dilation compression in time, it decomposes the signal into various scales corresponding to distinct frequency patterns.

\section{B. Development of Decision Tree based classifier}

Because of its properties pertaining to faster implementation and performance gains in bigger datasets, Decision Tree (DT) has been a prominent data mining technique for various classification problems in recent years. However, the prediction of DT is typically inferior to that of other data mining technologies such as SVM and deep neural networks. For complicated datasets, DT frequently fails to produce adequate testing results. Rather of employing a single DT for a specific classification task, the tree's ensemble is employed to tackle this problem [17]. The constraint associated with probable bias by a single DT is breached in order for the DT's output to be incorporated to the ultimate result. The

The flowchart presented in Figure 2 depicts the entire operation of the protection system based on DWT and Decision tree. As previously mentioned, the usable feature set produced via DWT is supplied to the Decision tree-based classifier, which is trained to achieve the maximum classification accuracy. For the PV integrated microgrid, separate Decision tree-based modules have been designed to execute the specialized tasks of fault detection and classification. Different instances containing large variations in fault and no-fault characteristics have been produced in each part of the distribution line. Variation in fault resistance, fault inception angle, and position were examined when creating scenarios involving line faults, whereas numerous random locations were explored when creating scenarios using PV array string faults such as stringstring and string to ground faults. The load fluctuation was taken into account while creating the no-fault situations

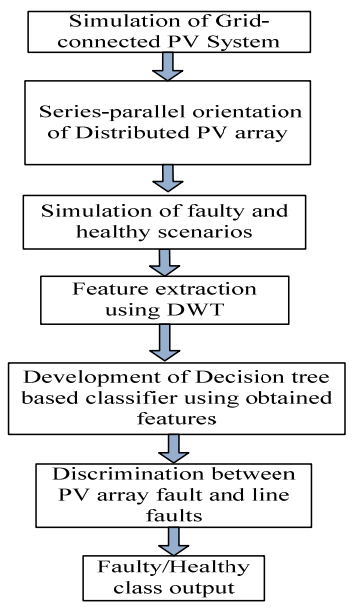

Figure 2: Flowchart of DWT and Decision tree based protection algorithm

The trained Decision tree-based module is examined for unknown test scenarios containing situations that were not taken into account during training.

\section{PERFORMANCE EVALUATION}

As discussed earlier, the Discrete wavelet transform extracts the essential information from current and voltage data acquired as a result of various sorts of faults in the distribution line and PV array. This data is further utilized to train a decision tree classifier to determine the sort of failure that has occurred in the system. A large data set is developed in the proposed study to train and 
International Research Journal of Engineering \& Applied Sciences, IRJEAS

www.irjeas.org, ISSN(O): 2322-0821, Volume 9 Issue 4, Oct-Dec 2021, Page 28-33

DOI - 10.55083/irjeas.2021.v09i04007 test the data-mining model (using Decision trees) in order to construct an accurate and robust classifier for performing the fault detection and classification job. The Decision tree model is trained and evaluated on a variety of data sets, including fault characteristics and other operational circumstances. For example, in a (70-30) data set, $70 \%$ of the data is used for training and $30 \%$ is used for testing.

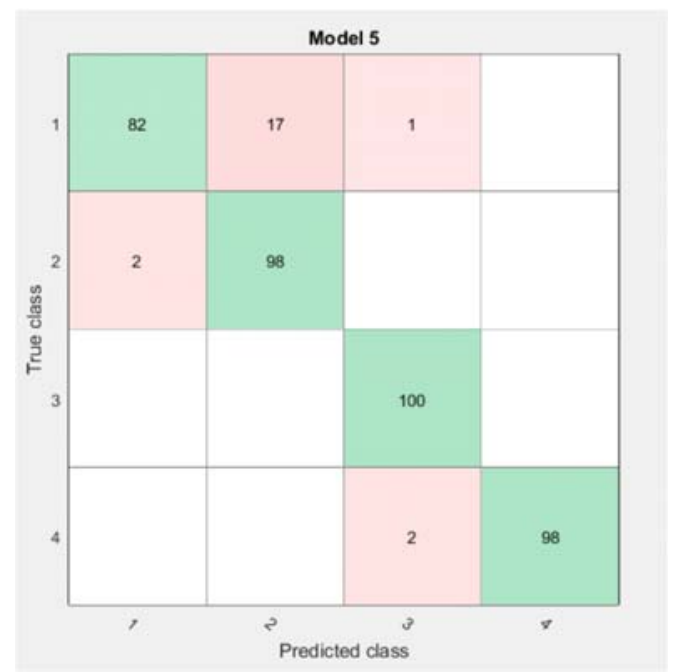

Figure 3: Confusion matrix showing the comparison between true and predicted class

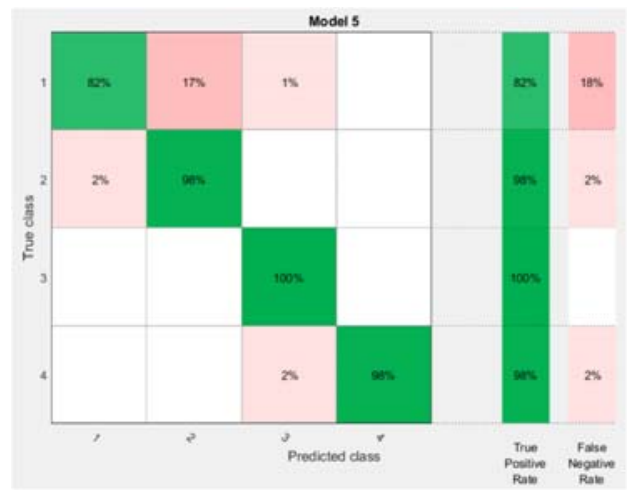

Figure 4: Confusion matrix showing the variation between true positive and true negative rate

In order to show the robustness of proposed bagged decision tree based classifier in performing the protection task, the Receiver Operating Characteristic (ROC) curve has been depicted in Figure 6 which shows the ability in performing the intended tasks.

In order to examine the performance of proposed DWT-Decision tree based proposed protection scheme over other classifier, a comparative assessment has been carried out with Support Vector Machine (SVM) and k-

The confusion matrix generated for the above system to demonstrate the training performance of proposed classifier is depicted in figures 3, 4 and 5, which provides the comparison results between the actual and predicted faults during testing for the given data set. The class labels are defined as given below: ' 1 ' represents the nofault class. The class labels ' 2 ' and ' 3 ' represents the fault types 'string to string' and 'string to ground' in the PV array while class ' 4 ' represents the distribution line faults.

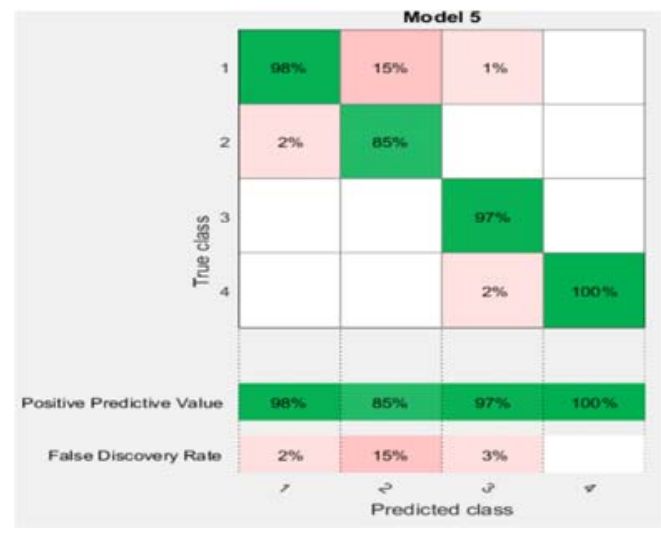

Figure 5: Confusion matrix showing the variation between positive predictive value and false discovery

rate

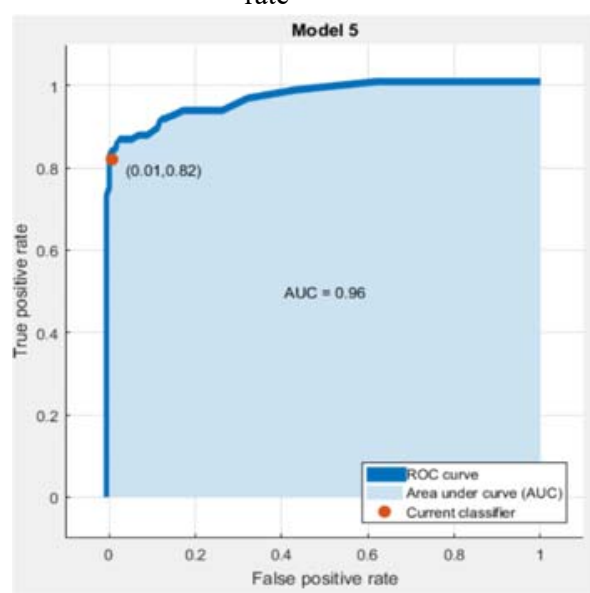

Figure 6: Receiver Operating Characteristic (ROC) curve of Bagged decision tree based classifier

nearest neighbour $(\mathrm{kNN})$ classifiers in Table I. For comparison, the total number of 500 test cases including 200 distribution line fault cases, 200 PV array fault cases and 100 no-fault cases were considered. The high classification accuracy of $98.6 \%$ achieved by proposed Decision tree based classifier over the SVM and kNN based classifier clearly confirms the efficacy of proposed approach in performing the intended protection task reliably. 
International Research Journal of Engineering \& Applied Sciences, IRJEAS

www.irjeas.org, ISSN(O): 2322-0821, Volume 9 Issue 4, Oct-Dec 2021, Page 28-33

DOI - 10.55083/irjeas.2021.v09i04007

Table I: Comparison of proposed Bagging tree classifier with other classifiers

\begin{tabular}{|c|c|c|c|c|c|c|}
\hline \multirow{2}{*}{ Type of classifier } & \multicolumn{4}{|c|}{ Number of test cases } & \multirow{2}{*}{$\begin{array}{c}\text { Correctly } \\
\text { predicted } \\
\text { Test } \\
\text { cases }\end{array}$} & \multirow{2}{*}{$\begin{array}{c}\text { Classification } \\
\text { Accuracy }\end{array}$} \\
\hline & Total & $\begin{array}{l}\text { Distribution } \\
\text { line faults }\end{array}$ & $\begin{array}{c}\text { PV } \\
\text { array } \\
\text { faults }\end{array}$ & $\begin{array}{l}\text { No- } \\
\text { faults }\end{array}$ & & \\
\hline Proposed Decision Tree & 500 & 200 & 200 & 100 & 493 & $98.6 \%$ \\
\hline $\begin{array}{l}\text { Support Vector Machine } \\
\text { (SVM) }\end{array}$ & 500 & 200 & 200 & 100 & 467 & $93.4 \%$ \\
\hline k Nearest Neighbour (kNN) & 500 & 200 & 200 & 100 & 451 & $90.2 \%$ \\
\hline & & & & & & \\
\hline
\end{tabular}

\section{CONCLUSION}

An efficient fault diagnosis scheme for Grid-connected PV systems has been proposed in this paper, which includes feature extraction using the Discrete wavelet transform, and classification of various defects on the PV system using Decision tree. The Grid-connected PV system under study has been simulated to generate the voltage-current signals with wide variation in the fault and other operating conditions and discriminatory attributes has been derived using DWT. The obtained

\section{REFERENCES}

[1] S. Dhar, R. K. Patnaik, and P. K. Dash, "Fault Detection and Location of Photovoltaic Based DC Microgrid Using Differential Protection Strategy," IEEE Trans. Smart Grid, vol. 3053, no. c, pp. 1-1, 2017.

[2] S. Bacha, D. Picault, B. Burger, I. Etxeberria-Otadui, and J. Martins, "Photovoltaics in microgrids: An overview of grid integration and energy management aspects," IEEE Ind. Electron. Mag., vol. 9, no. 1, pp. $33-46,2015$

[3] T. Abdelgayed, W. Morsi, and T. Sidhu, "A New Approach for Fault Classification in Microgrids Using Optimal Wavelet Functions Matching Pursuit," IEEE Trans. Smart Grid, vol. 3053, no. c, pp. 1-1, 2017.

[4] M. Manohar, E. Koley, and S. Ghosh, "Microgrid protection under weather uncertainty using joint probabilistic modeling of solar irradiance and wind speed R," Comput. Electr. Eng., vol. 86, p. 106684, 2020 .

[5] S. B. A. Bukhari, M. Saeed Uz Zaman, R. Haider, Y.S. Oh, and C.-H. Kim, "A protection scheme for microgrid with multiple distributed generations using superimposed reactive energy," Int. J. Electr. Power Energy Syst., vol. 92, pp. 156-166, 2017.

[6] A. Hooshyar and E. F. El-Saadany, "Fault Type Classification in Microgrids Including Photovoltaic DGs," IEEE Trans. Smart Grid, vol. 7, no. 5, pp. 2218-2229, 2016.

[7] D. Sampath Kumar, D. Srinivasan, and T. Reindl, "A attributes are further fed as input to the decision tree based classifier for the detection of faults in the PV array and distribution lines. The proposed protection strategy has been examined against wide number of unseen scenarios and compared with other classifiers including SVM and kNN based classifiers. The higher classification accuracy of $98.6 \%$ achieved using the proposed Decision tree based classifier over the SVM and $\mathrm{kNN}$ based classifier clearly confirms the efficacy of proposed approach in performing the intended protection task reliably.

Fast and Scalable Protection Scheme for Distribution Networks with Distributed Generation," IEEE Trans. Power Deliv., vol. PP, no. 99, pp. 1-1, 2015.

[8] M. Manohar, E. Koley, and S. Ghosh, "Reliable protection scheme for PV integrated microgrid using an ensemble classifier approach with real-time validation," IET Sci. Meas. Technol., vol. 12, no. 2, pp. 200-208, 2018.

[9] K. O. Oureilidis, C. S. Demoulias, and S. Member, "A Fault Clearing Method in Converter-Dominated Microgrids With Conventional Protection Means," i, vol. 31, no. 6, pp. 4628-4640, 2016.

[10] S. Dhar and P. K. Dash, "Differential current-based fault protection with adaptive threshold for multiple PV-based DC microgrid," IET Renew. Power Gener., vol. 11, no. 6, pp. 778-790, 2017.

[11] M. Sabbaghpur Arani and M. A. Hejazi, "The comprehensive study of electrical faults in PV arrays," J. Electr. Comput. Eng., vol. 2016, 2016.

[12] P. Ray and D. Prasad, "Engineering Science and Technology, an International Journal Support vector machine based fault classification and location of a long transmission line," Eng. Sci. Technol. an Int. J., vol. 19 , no. 3, pp. 1368-1380, 2016.

[13] D. P. Mishra, S. R. Samantaray, and G. Joos, "A Combined Wavelet and Data-Mining Based Intelligent Protection Scheme for Microgrid," IEEE Trans. Smart Grid, vol. 7, no. 5, pp. 2295-2304, 2016.

[14] S. Kar, "A comprehensive protection scheme for micro-grid using fuzzy rule base approach," Energy 
International Research Journal of Engineering \& Applied Sciences, IRJEAS

www.irjeas.org, ISSN(O): 2322-0821, Volume 9 Issue 4, Oct-Dec 2021, Page 28-33

Syst., 2016.

[15] S. Kar, S. R. Samantaray, and M. D. Zadeh, "DataMining Model Based Intelligent Differential Microgrid Protection Scheme," IEEE Syst. J., vol. PP, no. 99, pp. 1-9, 2015.

[16] A. Gururani and S. R. Mohanty, "Microgrid protection using Hilbert-Huang transform based-differential scheme," IET Gener. Transm. Distrib., vol. 10, no. 15, pp. 3707-3716, 2016.

DOI - 10.55083/irjeas.2021.v09i04007

[17] R. Polikar, "Ensemble based systems in decision making," IEEE Circuits Syst. Mag., vol. 6, no. 3, pp. 21-45, 2006.

[18] S. Kotsiantis, "Combining bagging, boosting, rotation forest and random subspace methods," Artif. Intell. Rev., vol. 35, no. 3, pp. 223-240, 2011. 